Research Article

\title{
Parkinson's Disease Motor and Non-Motor Features Accompanying Insomnia and Excessive Daytime Sleepiness Symptoms, a Large Population-Based Study
}

Aline Duarte Folle ${ }^{1}$, Kimberly C Paul ${ }^{1}$, Cynthia D Kusters ${ }^{1,2}$, Jeff M Bronstein ${ }^{3}$, Adrienne M Kenner 3,4 , Beate Ritz $1,3, *$

1. Department of Epidemiology, UCLA Fielding School of Public Health, Los Angeles, California, USA; E-Mails: alinefolle@ucla.edu; kimberlyc.paul@gmail.com; cynthiakusters@gmail.com; britz@ucla.edu

2. Department of Human Genetics, UCLA David Geffen School of Medicine, Los Angeles, California, USA

3. Department of Neurology, UCLA David Geffen School of Medicine, Los Angeles, California, USA; E-Mails: JBronste@mednet.ucla.edu; AKeener@mednet.ucla.edu

4. Department of Neurology, Veterans Administration Greater Los Angeles Healthcare System, Los Angeles, California, USA

* Correspondence: Beate Ritz; E-Mail: britz@ucla.edu

Academic Editor: Roy G. Beran

Special Issue: Sleep Disorders in the Elderly

OBM Geriatrics

2020, volume 4 , issue 3

doi:10.21926/obm.geriatr.2003131
Received: June 30, 2020

Accepted: August 18, 2020

Published: August 26, 2020

\begin{abstract}
Insomnia and excessive daytime sleepiness are the most common sleep disturbances in Parkinson's disease. This study aims at better understanding how severity of PD motor and non-motor features and dopaminergic treatments contribute to these sleep symptoms in the first decade of PD. Data from a community-based cohort of PD patients was used to model cross-sectional PD-related risk factors for insomnia and EDS sleep scores using linear regression models adjusted for age, gender, and PD duration. Longitudinal changes in sleep scores were assessed with paired t-tests. For 481 patients who completed the MOS-Sleep
\end{abstract}

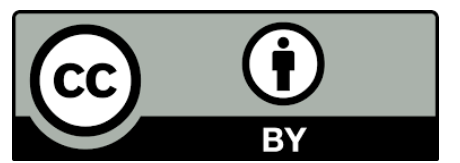

(C) 2020 by the author. This is an open access article distributed under the conditions of the Creative Commons by Attribution License, which permits unrestricted use, distribution, and reproduction in any medium or format, provided the original work is correctly cited. 
questionnaire at least once, high levodopa daily doses (500mg+) and severe autonomic and complex non-motor symptoms (depression, anxiety, apathy, hallucinations and dopamine dysregulation syndrome) were associated with both EDS and insomnia symptoms. Higher total motor UPDRS and especially tremor sub-scores and motor complications were associated only with insomnia, while axial/posture/gait and body bradykinesia UPDRS sub-scores were associated only with EDS. In 156 patients, with a second sleep measure on average after 2.2 years of follow-up, only EDS scores increased over time. Groups defined by worse PD features severity at first follow-up (UPDRS 35+, PD duration 6.5+ years, or LED 500mg+) had larger average increases in EDS score over time. These findings provide evidence that motor and non-motor dysfunction in PD are associated with insomnia and EDS symptoms, but specific features and level of severity affect sleep symptoms differently. Motor manifestations related to tremor and dyskinesia are associated with sleep quantity and quality, measured by insomnia symptoms, while axial motor features are related to EDS symptoms.

\section{Keywords}

Parkinson's disease; motor symptoms; non-motor symptoms; sleep problems; circadian dysfunction; insomnia; excessive daytime sleepiness

\section{Introduction}

Sleep disturbances have been increasingly recognized as important non-motor components of the Parkinson's disease (PD) syndrome [1-5]. The most frequent sleep or sleep-related problems that patients report are excessive daytime sleepiness (EDS), insomnia and REM sleep behavior disorder (RBD) [6]. Insomnia and EDS are sleep-wake disturbances that may indicate disruption of the circadian rhythm as part of the neurodegenerative process characteristic of PD $[7,8]$.

Insomnia is the difficulty to initiate or maintain sleep and, when it manifests chronically, insomnia has well-known negative consequences for health and quality of life $[9,10]$. While PD patients usually do not have trouble initiating sleep, insomnia manifests mainly as the difficulty to maintain sleep, resulting in sleep fragmentation and early awakening [11]. EDS refers to a subjective complaint characterized by difficulty in remaining awake during the day, usually accompanied by sleep initiation if the person stays inactive [12], and it is more common in PD patients than in the general population [13]. EDS can be secondary to other sleep disorders and health factors, or can be attributable to primary central disorders of hypersomnolence, as defined in the International Classification of Sleep Disorders (ICSD) [14].

Better insight into what causes sleep problems in PD will encourage improvements in clinical care and quality of life of patients. In the last decade, a number of studies suggested the following risk factors for insomnia and/or EDS in PD [15-24]: PD duration, motor disability, dopaminergic medications, depression and anxiety, and autonomic symptoms. However, these studies mainly enrolled patients from tertiary clinics or, when community-based, they were conducted in European countries or had a small number of participants. Also, they yielded conflicting results as to which PD-related clinical symptoms are related to sleep disorders. Previously, data from a large community-based cohort of PD patients was analyzed to investigate the role of REM sleep behavior 
disorder on PD progression [25]. Relying on the same cohort of PD patients, the present study assesses contributions of severity of PD motor and non-motor features and treatment with levodopa to insomnia and EDS symptoms in patients who are, on average, within six years (range: 2 - 15) of an initial PD diagnosis.

\section{Methods}

\subsection{Study Design}

The Parkinson's Environment and Genes Study (PEG), identified new-onset (up to 5 years after diagnosis) PD cases at baseline from 2001 to 2007 (PEG 1), and from 2011 to 2017 (PEG 2), from the entire population of three California counties [26]. PEG $1 \& 2$ participants were seen for a first followup, on average 3.2 years after their baseline visit. PEG 1 participants were additionally seen a second time, on average $2.2( \pm 0.5)$ years later. At all time points, participants were examined at a local clinic by PEG study movement disorders specialists, who confirmed the diagnosis according to common criteria [26], and evaluated motor signs and symptoms, preferably with patients "off" PD medications.

The UCLA Institutional Review Board approved all phases of the study protocol, and participants were informed of all procedures and their rights and provided written informed consent.

\subsection{Data Collected}

Study neurologists examined patients and scored motor disability using the Unified Parkinson's Disease Rating Scale (UPDRS, and later MDS-UPDRS), parts III and IV and Hoehn and Yahr staging (HY). The exams were preferably conducted with patients "off" PD medications (>90\% of exams were "off"). UPDRS-III scores were corrected for missing items that cannot be evaluated (such as arising from chair in paraplegic patients), and when only an "on medication" exam was possible, as previously described [27]. PD motor subtypes of Postural Imbalance and Gait Dysfunction (PIGD), Tremor Dominant (TD), or indeterminate were calculated as ratios of UPDRS-III sub-scores, as described previously [28]. PD medication information, including levodopa and dopamine agonist use, were summarized into a daily levodopa equivalent dose (LED) [29].

During all visits, trained research assistants interviewed participants to collect demographic, lifestyle, and medical history information, including current PD medication use and dosage. We calculated UPDRS-III motor total score and sub-scores; the latter by summing specific items corresponding to total tremor [30] (rest and postural), rigidity, limb bradykinesia [31] (fingers tapping, hands grip, hands rapid movements, leg agility), and axial/posture/gait [31] (speech, facial expression, arise from chair, posture, postural stability, gait, and body bradykinesia).

Additional standardized instruments were adopted only during follow-up, including those measuring insomnia and EDS, as well as the UPDRS-IA, IB and II to assess non-motor and motor impacts of PD on experiences of daily living. We rely on the Sleep Survey of the Medical Outcomes Study (MOS-Sleep) for recording symptoms of insomnia and EDS. It contains twelve items, each with six answer options on a Likert scale, measuring subjective experiences of sleep in the past four weeks across several domains including sleep initiation, maintenance, quantity/duration, perceived adequacy, respiratory problems and somnolence. The MOS-Sleep has been validated and been used to study chronic diseases; its content is very comparable to two questionnaires widely used in PD 
sleep research, the Pittsburgh Sleep Quality Index (PSQI) and the Parkinson's Disease Sleep Scale (PDSS) [32].

MOS-Sleep items are summarized to create five scores (sleep disturbance, somnolence, sleep adequacy, snoring, and shortness of breath during sleep) ranging from 0 to 100, with higher scores indicating worse sleep quality, except for the adequacy score, which is reversed. For this study's purposes, we adopted the continuous scores (0 to 100) for sleep disturbance (items: having trouble falling asleep, how long to fall asleep, sleep was not quiet, awake during sleep time, and having trouble falling asleep again) as a measure of insomnia symptoms, and for somnolence (items: drowsy during day, having trouble staying awake during the day, taking naps), as a measure of EDS symptoms.

\subsection{Statistical Analysis}

Analyses were conducted in statistical software SAS (SAS 9.4, SAS Institute, Cary, NC), figures were generated in R. Insomnia and EDS scores were normally distributed univariately and across risk factors of interest; such as gender, age and PD duration at the time sleep measures.

Sleep scores were $z$-standardized, centering on mean 0 and standard deviation 1 . First, mean crude z-standardized MOS-Sleep scores by patients' characteristics were estimated. To visualize how PD-related factors (PD duration, age at diagnosis, UPDRS-III, UPDRS-IA, and LED) may influence sleep symptoms, crude z-standardized insomnia and EDS scores were plotted according to these PD-related factors, stratifying by gender, and generating Pearson correlation coefficients.

Adjusted mean differences in sleep scores between binary measures of PD severity were modeled using linear regressions (implemented with maximum likelihood in Proc Genmod; SAS 9.4) including potential confounders as covariates; i.e., at a minimum we included gender, age at interview, and PD duration. PD measures were dichotomized, i.e., at the median for PD duration (6.5 years) at first sleep assessment; at 35 points for the UPDRS-III motor; at $500 \mathrm{mg}$ for the LED as proposed previously [17]; for the UPDRS-II (20 points), UPDRS-IA (5 points), and the autonomic symptoms score ( 8 points) the cut-points correspond to the respective $75^{\text {th }}$ percentile of the score. To estimate associations between UPDRS motor sub-scores with insomnia and EDS scores, similar adjusted linear regression models were used, but continuous motor sub-scores were also zstandardized and results presented as $\beta$-coefficients. In sensitivity analyses, additional potential confounders were included guided by mechanisms proposed and depicted in Directed Acyclic Graphs [33] (Supplemental Figure S1) as detailed in Results.

In a subset of the PEG1 cohort with information on sleep measures $(n=156)$ at an additional follow-up time, average sleep scores at both follow-up times stratified by PD severity measures were compared using t-tests as well as paired t-tests to identify differences in average sleep scores over time by PD-severity at first follow-up (between and within groups).

\section{Results}

This study included 481 PD patients who completed the MOS-Sleep at least once, of whom 459 (95\%) also completed a simultaneous motor examination. The majority was male (62\%), White (77\%), assessed for sleep quality on average $6.3 \pm 3.0$ years after their first PD diagnosis, and $69 \%$ exhibited a PIGD motor phenotype (Table 1). Among the sleep domains the MOS assesses, EDS received the highest absolute score, on a scale from 0 to 100 (mean $42.4 \pm$ SD 23.7), followed by 
snoring (34.5 \pm 33.4$)$ and insomnia $(30.5 \pm 22.6)$. Insomnia and EDS measures were moderately positively correlated $(\rho=0.34$ ) (Supplemental Figure S2). Figure 1 shows linear correlations of MOSSleep scores with PD-related measures; patients diagnosed at younger ages and with longer PD duration had worse insomnia symptom scores, but these PD features were not correlated with EDS (Figure 1). All other PD-related measures were positively correlated with both insomnia and EDS, in men and women.

Table 2 presents cross-sectional mean differences in insomnia and EDS scores comparing groups defined by PD severity, adjusting for gender, age and PD duration. In these models, a PD duration of 6.5+ years was associated with higher EDS, but not insomnia. Conversely, a UPDRS-III motor total score of $35+$ and the presence of motor complications were associated with higher average insomnia scores, but not with EDS. Specifically, off-dystonia and motor fluctuations were the motor complications associated with worse insomnia scores. Estimates for motor complications remained unchanged in models further adjusted for levodopa use or dose, and for UPDRS-III scores. Likewise, the strong positive associations of non-motor (UPDRS-IA) and autonomic symptoms with insomnia and EDS scores persisted when the models were further adjusted for UPDRS-III or levodopa dose.

Only few patients did not take PD medications (8\%) or solely used dopamine agonists (6\%), while $54 \%$ were treated with levodopa only, and $32 \%$ with a combination of levodopa and dopamine agonists (Table 1). Patients with LED $\geq 500 \mathrm{mg}$ had worse insomnia and EDS symptoms, compared to the group with $<500 \mathrm{mg}$, while higher doses of dopamine agonists ( $\geq 200 \mathrm{mg} v \mathrm{vs}$. $<200 \mathrm{mg}$ ) did not significantly impact the sleep scores. These estimates remained unchanged in models that included the motor UPDRS-III scores, or axial/postural/gait or tremor sub-scores (results not shown).

Table 1 Distribution of demographics, PD-related characteristics and MOS-Sleep scale scores for Insomnia and EDS, at first follow-up.

\begin{tabular}{|c|c|c|c|c|}
\hline & & \multirow{2}{*}{ N (\%) } & \multicolumn{2}{|c|}{$\begin{array}{l}\text { Crude Mean Standardized MOS- } \\
\text { Sleep score }\end{array}$} \\
\hline & & & $\begin{array}{c}\text { Insomnia } \\
\text { (Mean } \pm \text { SD) }\end{array}$ & $\begin{array}{c}\text { EDS } \\
(\text { Mean } \pm \text { SD) }\end{array}$ \\
\hline \multicolumn{2}{|l|}{ Total } & $481(100)$ & $0 \pm 1$ & $0 \pm 1$ \\
\hline \multirow[t]{2}{*}{ Study cohort ${ }^{1}$} & PEG 1 & 234 (49) & $-0.051 \pm 0.978$ & $0.034 \pm 0.978$ \\
\hline & PEG 2 & $247(51)$ & $0.049 \pm 1.020$ & $-0.033 \pm 1.022$ \\
\hline \multirow[t]{3}{*}{ Age at interview } & 65 or less & $119(25)$ & $0.299 \pm 1.139$ & $0.040 \pm 1.026$ \\
\hline & 66 to 80 & $263(55)$ & $-0.107 \pm 0.895$ & $-0.013 \pm 0.937$ \\
\hline & more than 80 & $95(20)$ & $-0.078 \pm 1.026$ & $-0.013 \pm 1.136$ \\
\hline \multirow[t]{2}{*}{ Gender } & women & $183(38)$ & $0.002 \pm 0.993$ & $-0.226 \pm 0.942$ \\
\hline & men & $298(62)$ & $-0.001 \pm 1.006$ & $0.141 \pm 1.010$ \\
\hline \multirow[t]{3}{*}{ Ethnicity } & White & $371(77)$ & $-0.057 \pm 0.959$ & $0 \pm 0.985$ \\
\hline & Latino & $79(17)$ & $0.183 \pm 1.112$ & $-0.059 \pm 1.060$ \\
\hline & Other & $29(6)$ & $0.228 \pm 1.124$ & $0.160 \pm 1.043$ \\
\hline \multirow[t]{2}{*}{ Education } & $<12$ years & $71(15)$ & $0.259 \pm 1.097$ & $-0.037 \pm 1.064$ \\
\hline & $12+$ & $411(85)$ & $-0.045 \pm 0.978$ & $0.009 \pm 0.989$ \\
\hline \multirow[t]{2}{*}{ Age at PD diagnosis } & $\leq 60$ & $131(27)$ & $0.284 \pm 1.136$ & $0.089 \pm 1.034$ \\
\hline & $>60$ & $350(73)$ & $-0.104 \pm 0.925$ & $-0.033 \pm 0.987$ \\
\hline
\end{tabular}




\begin{tabular}{|c|c|c|c|c|}
\hline \multirow[t]{2}{*}{ Age at PD duration } & $\leq 6.5$ years & $279(58)$ & $-0.120 \pm 0.906$ & $-0.131 \pm 0.948$ \\
\hline & $>6.5$ years & $202(42)$ & $0.168 \pm 1.099$ & $0.183 \pm 1.043$ \\
\hline \multirow[t]{2}{*}{$\begin{array}{l}\text { Motor subtype } \\
\text { (missing=27) }\end{array}$} & $\begin{array}{l}\text { tremor dominant or } \\
\text { indeterminate }\end{array}$ & $151(33)$ & $-0.032 \pm 0.939$ & $-0.074 \pm 0.969$ \\
\hline & PIGD & $303(67)$ & $0.030 \pm 1.025$ & $0.035 \pm 0.990$ \\
\hline \multirow{2}{*}{$\begin{array}{l}\text { UPDRS-III2 total } \\
\text { score }\end{array}$} & $<35$ (missing $=22$ ) & $349(76)$ & $-0.054 \pm 0.957$ & $-0.041 \pm 0.951$ \\
\hline & $35+$ & $110(24)$ & $0.235 \pm 1.107$ & $0.146 \pm 1.082$ \\
\hline \multirow[t]{2}{*}{ Hoehn and Yahr } & stages $0-2.5($ missing $=33)$ & $330(74)$ & $-0.011 \pm 0.973$ & $-0.063 \pm 0.957$ \\
\hline & stage $3+$ & $118(26)$ & $-0.025 \pm 1.054$ & $0.105 \pm 0.991$ \\
\hline \multicolumn{2}{|c|}{ LED 3 Dopamine agonists only, 200 mg+ } & $114(25)$ & $0.036 \pm 1.001$ & $0.009 \pm 0.929$ \\
\hline \multicolumn{2}{|c|}{ LED ${ }^{3}$ Levodopa only, 500 mg+ } & $188(40)$ & $0.164 \pm 1.064$ & $0.240 \pm 1.046$ \\
\hline \multicolumn{2}{|l|}{ LED $^{3}$ total, $600 \mathrm{mg}^{+}$} & $197(42)$ & $0.154 \pm 1.063$ & $0.146 \pm 1.039$ \\
\hline \multicolumn{2}{|c|}{$\begin{array}{l}\text { UPDRS-IV }{ }^{4} \text { (motor complications) any present } \\
\text { (missing }=22 \text { ) }\end{array}$} & $235(53)$ & $0.200 \pm 1.051$ & $0.041 \pm 0.923$ \\
\hline \multirow[t]{3}{*}{ UPDRS-IV sub-scores } & Dyskinesia, present ${ }^{5}$ & $97(22)$ & $0.259 \pm 1.027$ & $0.031 \pm 0.896$ \\
\hline & $\begin{array}{l}\text { Motor Fluctuations, } \\
\text { present }\end{array}$ & $190(43)$ & $0.223 \pm 1.036$ & $0.015 \pm 0.941$ \\
\hline & Off-Dystonia, present & 77 (17) & $0.380 \pm 1.119$ & $0.183 \pm 0.956$ \\
\hline \multicolumn{2}{|c|}{ UPDRS-II ${ }^{6}$ (motor ADL) score, 20+ } & $140(32)$ & $0.269 \pm 1.092$ & $0.305 \pm 1.011$ \\
\hline \multirow{3}{*}{$\begin{array}{l}\text { UPDRS-IA (non- } \\
\text { motor, complex } \\
\text { behaviours) }\end{array}$} & score $5+$ & $114(25)$ & $0.450 \pm 1.121$ & $0.359 \pm 0.974$ \\
\hline & $\begin{array}{l}\text { Autonomic sympoms }{ }^{8} \text {, } \\
\text { score } 8+\end{array}$ & $118(25)$ & $0.177 \pm 1.051$ & $0.331 \pm 0.959$ \\
\hline & Urinary problems, present & $350(74)$ & $0.070 \pm 1.013$ & $0.100 \pm 0.999$ \\
\hline
\end{tabular}

1. PEG=Parkinson's Environment and Genes.

2. UPDRS-III (rated by physician), motor signs: speech, facial expression, tremor at rest (face, hands, feet) amplitude and constancy, rigidity (neck, arms, legs), fingers and toes tapping, hand grip and movements, leg agility, arising from chair, posture, gait and freezing of gait, postural stability, body bradykinesia, postural and kinetic tremor.

3. LED: Levodopa Equivalent Daily Dose.

4. UPDRS-IV (applied by physician), motor complications items: dyskinesias (time spent and functional impact), motor fluctuations (time spent in off-state, functional impact and complexity of fluctuations), painful off-state present and time spent.

5. Presence of dyskinesia: measured by UPDRS-IV question "Time Spent with Dyskinesias", where option " $0=$ Normal" corresponds to "No Dyskinesia" and any other option (1,2,3 or 4) corresponds to "Yes Dyskinesia".

6. UPDRS-II: Motor Aspects of Experiences of Daily Living (self-completed) items: speech, saliva/drooling, chewing/swallowing, eating, dressing, hygiene, handwriting, hobbies, turning in bed, tremor, getting off bed/car/chair, walking/balance, freezing.

7. UPDRS-I: Non-Motor Aspects of Experiences of Daily Living.

8. UPDRS-IA (rated by physician), complex behaviours items: cognitive impairment, hallucinations, depressed mood, anxious mood, apathy, features of dopamine dysregulation syndrome.

9. Autonomic symptoms score: constipation, urinary, light headedness, saliva/drooling, chewing/swallowing. 

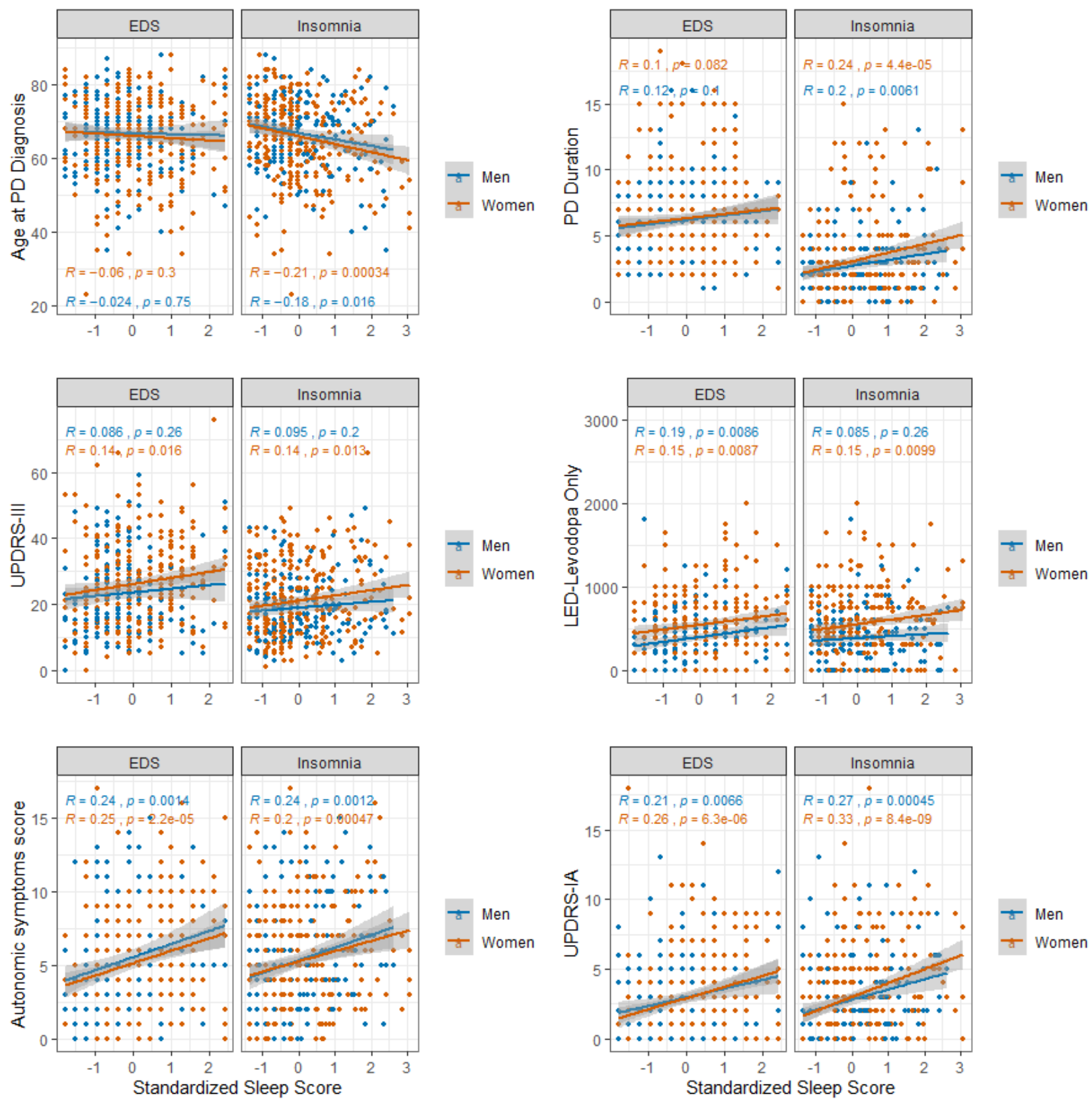

Figure 1 Scatterplot of standardized sleep scores (mean 0, SD 1) by PD characteristics stratified by gender. Including best fit linear correlation line, Pearson correlation coefficients, and $\mathrm{p}$-values. $\mathrm{N}=481$ (except for UPDRS-III, $\mathrm{N}=459$ ). 
Table 2 Linear regressions of insomnia and EDS scores on PD-related characteristics (cross-sectional), adjusted for gender, age and duration of Parkinson's disease in years, $\mathrm{N}=459$.

\begin{tabular}{|c|c|c|}
\hline \multirow{2}{*}{ PD severity measures (binary) } & Insomnia & EDS \\
\hline & \multicolumn{2}{|c|}{ Mean difference ${ }^{1}(95 \% \mathrm{Cl})$} \\
\hline PD duration ${ }^{2}$, years, $6.5+$ vs. $<6.5$ & $0.02(-0.26,0.29)$ & $0.34(0.06,0.62)$ \\
\hline UPDRS-III ${ }^{3}$ total score, $35+$ vs. $<35$ & $0.27(0.06,0.49)$ & $0.14(-0.07,0.35)$ \\
\hline HY, stage $3+$ vs. stage $<3($ Total $N=448)$ & $0.08(-0.13,0.29)$ & $0.19(-0.02,0.40)$ \\
\hline Motor subtype, PIGD ${ }^{4}$ vs. other & $0.12(-0.07,0.32)$ & $0.14(-0.05,0.33)$ \\
\hline LED $^{5}$, Levodopa only, $500 \mathrm{mg}+$ vs. $<500 \mathrm{mg}$ & $0.22(0.03,0.41)$ & $0.29(0.10,0.47)$ \\
\hline Only dopamine agonists, $200 \mathrm{mg}+\mathrm{vs} .<200 \mathrm{mg}$ & $-0.10(-0.32,0.11)$ & $-0.05(-0.26,0.17)$ \\
\hline Total, Levodopa and dopamine agonists, $600 \mathrm{mg}+$ vs. $<600 \mathrm{mg}$ & $0.17(-0.02,0.35)$ & $0.15(-0.04,0.34)$ \\
\hline UPDRS-IV 6 total (motor complications), any present vs. not & $0.25(0.06,0.44)$ & $0.04(-0.15,0.23)$ \\
\hline UPDRS-IV, Dyskinesia7, present vs. not & $0.13(-0.10,0.36)$ & $-0.04(-0.27,0.19)$ \\
\hline Motor Fluctuations, present vs. not & $0.24(0.05,0.43)$ & $-0.02(-0.20,0.17)$ \\
\hline Off-Dystonia, present vs. not & $0.33(0.09,0.58)$ & $0.23(-0.01,0.47)$ \\
\hline UPDRS- $\|^{8}$ score, $20+$ vs. $<20$ & $0.37(0.18,0.57)$ & $0.40(0.20,0.60)$ \\
\hline UPDRS-IA ${ }^{9}$ score, $8+$ vs. $<8$ & $0.54(0.34,0.74)$ & $0.45(0.25,0.65)$ \\
\hline Autonomic symptoms ${ }^{10}$ score, $5+$ vs. $<5$ & $0.27(0.06,0.48)$ & $0.43(0.23,0.64)$ \\
\hline Urinary problems, present vs. not & $0.27(0.07,0.47)$ & $0.32(0.12,0.52)$ \\
\hline
\end{tabular}

1. Adjusted for gender, age, and duration of PD.

2. Adjusted for gender and age.

3. UPDRS-III (rated by physician), motor signs: speech, facial expression, tremor at rest (face, hands, feet) amplitude and constancy, rigidity (neck, arms, legs), fingers and toes tapping, hand grip and movements, leg agility, arising from chair, posture, gait and freezing of gait, postural stability, body bradykinesia, postural and kinetic tremor.

4. Postural Instability and Gait Disturbance (or Dysfunction, Difficulty)

5. LED: Levodopa Equivalent Daily Dose

6. UPDRS-IV (applied by physician), motor complications items: dyskinesias (time spent and functional impact), motor fluctuations (time spent in off-state, functional impact and complexity of fluctuations), painful off-state present and time spent.

7. Presence of dyskinesia: measured by UPDRS part IV question "Time spent with dyskinesias", where option "0=Normal" corresponds to "no dyskinesia" and any other option (1,2,3 or 4) corresponds to "yes Dyskinesia"

8. UPDRS-II (self-completed), motor aspects of experiences of daily living items: speech, saliva/drooling, chewing/swallowing, eating, dressing, hygiene, handwriting, hobbies, turning in bed, tremor, getting off bed/car/chair, walking/balance, freezing.

9. UPDRS-IA (rated by physician), complex behaviors items: cognitive impairment, hallucinations, depressed mood, anxious mood, apathy, features of dopamine dysregulation syndrome.

10. Autonomic symptoms score: constipation, urinary, light headedness, saliva/drooling, chewing/swallowing. 
Table 3 shows adjusted linear associations of UPDRS-III motor sub-scores with insomnia and EDS. Total tremor and limb bradykinesia were positively associated only with worse insomnia, while axial/postural/gait was associated only with EDS scores, however, these associations were greatly attenuated in models adjusted for (levodopa-only) LED (results not shown). The association of the body bradykinesia sub-score with EDS persisted after adjustment for LED, but was greatly reduced after adjustment for the geriatric depression scale (GDS) score $(\beta=0.06 ; 95 \% \mathrm{Cl}:-0.03,0.15)$.

Table 3 Linear regressions of insomnia and EDS scores on UPRDS-III motor sub-scores (cross-sectional), adjusted for gender, age and duration of Parkinson's disease in years. Sleep scores and motor sub-scores are z-standardized, $\mathrm{N}=459$.

\begin{tabular}{lcc}
\hline $\begin{array}{l}\text { UPDRS-III motor sub-scores, per } \\
\text { 1 SD increase }\end{array}$ & $\begin{array}{c}\text { Insomnia } \\
\boldsymbol{B} \text { Coefficient (95\% Cl) }\end{array}$ & $\boldsymbol{\beta}$ Coefficient (95\% Cl) \\
\hline Total tremor & $0.09(0.00,0.18)$ & $0.04(-0.05,0.13)$ \\
Limb bradykinesia & $0.11(0.02,0.21)$ & $0.06(-0.03,0.15)$ \\
Rigidity & $0.05(-0.04,0.15)$ & $-0.01(-0.11,0.08)$ \\
Axial, posture and gait & $0.07(-0.03,0.17)$ & $0.10(0.00,0.20)$ \\
Body bradykinesia & $-0.01(-0.10,0.09)$ & $0.10(0.01,0.19)$ \\
\hline
\end{tabular}

Longitudinal information on insomnia and EDS scores was available for 156 participants from the PEG1 study cohort at a second follow-up, on average $2.2 \pm 0.5$ years after the first, and an average PD duration of $7.4 \pm 2.5$ years (for the PEG 2 cohort a second follow-up has not yet been completed). Of the patients not seen for a second follow-up in PEG1, the majority (65\%) had died or were too ill, including cognitive dysfunction. Changes in average MOS-Sleep scores over time were minor. Overall, EDS scores slightly increased from first to second follow-up, the within-person average difference was $3.0(95 \% \mathrm{Cl}:-0.7,6.6)$ points on the non-standardized score, i.e., on a scale from 0 to 100 , whereas insomnia average scores did not change $(-1.4,95 \% \mathrm{Cl}:-4.6,1.8)$. Figure 2 shows average within-person changes in insomnia and EDS scores, by PD severity measures, with t-tests comparing scores between groups at each follow-up time. At first follow-up, the severity measures UPDRS $\geq$ vs. $<35$ and LED $\geq$ vs. $<500 \mathrm{mg}$ and a longer PD duration ( $\geq$ vs. $<6.5 \mathrm{y}$ ) were associated with worse concurrent insomnia scores, but these measures did not predict worsening of insomnia scores at the second follow-up. For EDS, on the other hand, higher PD severity and longer duration at first follow-up were associated with worse EDS scores at the second follow-up. 


\section{Insomnia and EDS Scores: Change Over Time}

UPDRS-III(motor) $\rightarrow<35-\bullet=35$

UPDRS-III(motor) $\rightarrow<35 \rightarrow-=35$
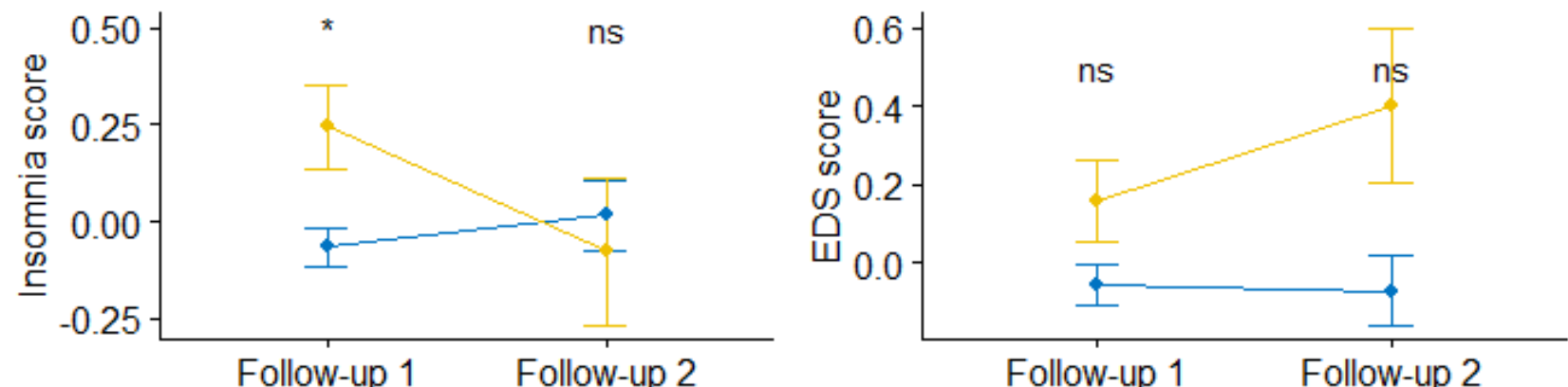

Follow-up $1 \quad$ Follow-up 2
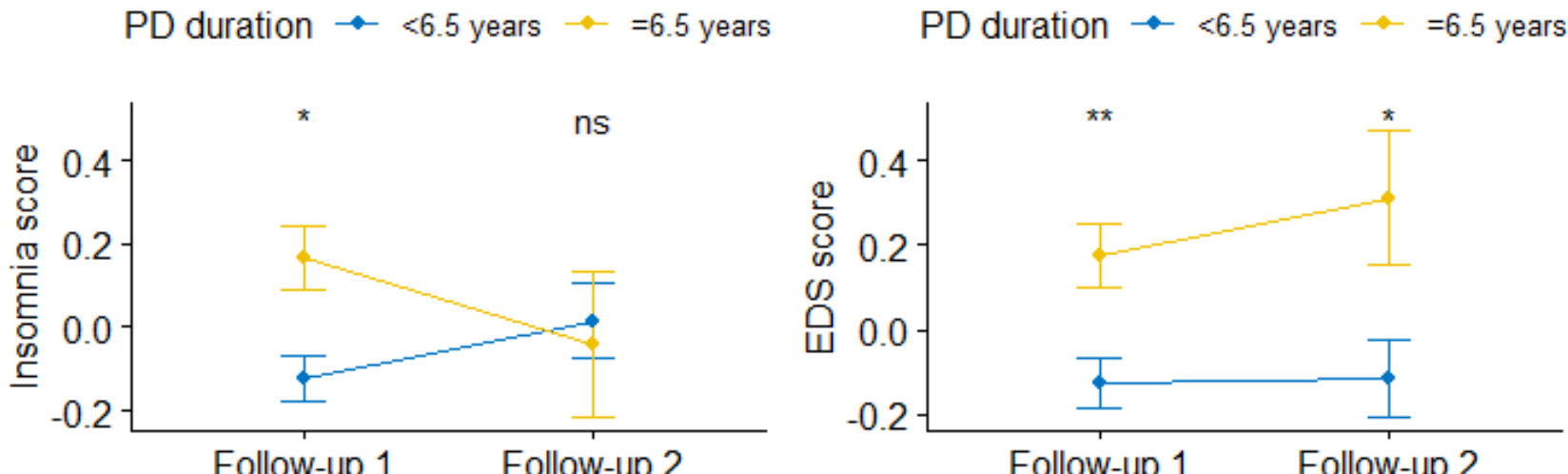

Follow-up 1

Follow-up $1 \quad$ Follow-up 2

LED(Levodopa) $\rightarrow<500 \mathrm{mg} \rightarrow-=500 \mathrm{mg}$

LED(Levodopa) $\rightarrow<500 \mathrm{mg} \rightarrow-=500 \mathrm{mg}$

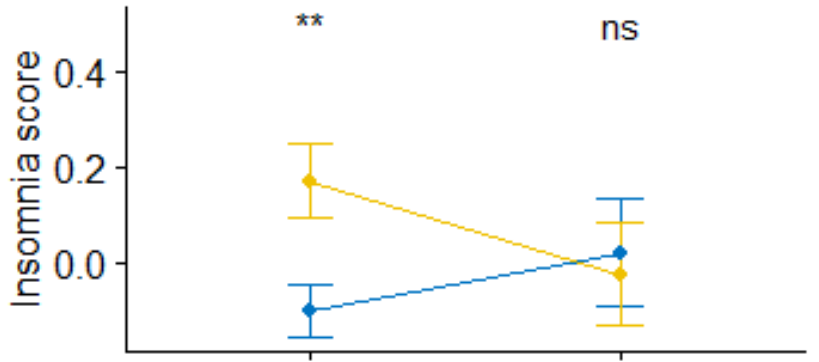

Follow-up $1 \quad$ Follow-up 2

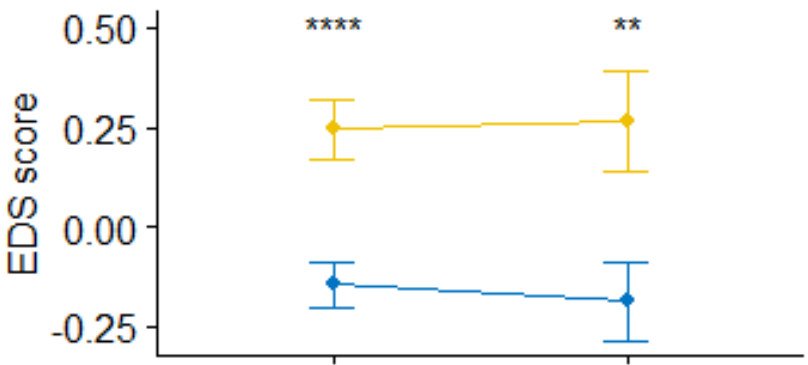

Follow-up $1 \quad$ Follow-up 2

Figure 2 Change in sleep scores from follow-up 1 to follow-up $2(\mathrm{~N}=156)$, by PD severity measures at follow-up 1 . Results of significance for t-test: ${ }^{*}=p<0.05, * *=p<0.01$, $* * * *=p<0.0001, n s=p>0.05$. 


\section{Discussion}

This large population-based study of Parkinson's disease assessed associations of PD clinical characteristics with insomnia and EDS symptoms among patients with on average six years of disease duration. For a subgroup of 156 patients, sleep information was collected again two years later. In this cohort, virtually all aspects of PD severity evaluated were associated either with worse insomnia, EDS, or with both sleep symptoms, confirming the burden of this non-motor problem during the progression of PD. Total motor UPDRS-III scores, sub-scores of tremor and limb bradykinesia, and UPDRS-IV motor fluctuations were associated with worse insomnia symptoms but not with EDS. Other aspects of PD, such as longer disease duration and motor sub-scores of axial/posture/gait and body bradykinesia were associated only with EDS symptoms. Levodopa doses $\geq 500 \mathrm{mg}$ and autonomic symptoms were associated with worse scores for both sleep problems. For the group with one additional follow-up, only EDS scores were slightly worse after 2.2 years on average, while insomnia scores remained similar.

Neurodegeneration of sleep-wake regulatory centers in PD resulting in circadian disruption [34] is one possible explanation for the association between worse total motor scores and insomnia. The finding that motor UPDRS total score and sub-scores of tremor, limb bradykinesia and motor fluctuations affect only insomnia symptoms can also indicate that these motor manifestations directly disrupt nighttime sleep. Since this study's patients are examined "off medication", those with high tremor sub-scores likely have worse tremor during off states at night. Worse tremor during night off states have been shown to lead to increased sleep fragmentation, resulting from re-emergence of resting tremor during micro-arousals, body movements and sleep-state changes (mainly from NREM to REM sleep) [35]. Similarly, limb bradykinesia during night off states may cause difficulties in turning and adjusting the body position in bed, which are problems known to cause sleep fragmentation in PD patients [11,36]. It has also been shown previously [8] that motor activity from tremor or motor fluctuations may disrupt the circadian system directly, since body movements impact the physiological cues used by this system.

Three larger studies that related motor disability to insomnia have been descriptive and none have analyzed motor UPDRS sub-scores, but they corroborate the present study's findings for positive associations of overall motor severity and insomnia symptoms. A large population-based French study (COPARK), reported cross-sectional results for 636 PD patients who responded to the PSQI questionnaire [18] with a mean PD duration similar to our study (6.3 years) and found higher motor UPDRS total scores in those with sleep disturbance (defined as PSQI score above 5, similar in content to the MOS-sleep insomnia measure adopted here), but the results reported were unadjusted for potential confounders, such as age, gender and PD duration. A population-based Norwegian cohort $(n=231)$ also reported insomnia to be associated with higher motor UPDRS total scores, but estimates from this study were not formally statistically significant and, again, unadjusted for potential confounders [17]. In addition, this population had a higher PD duration at time of study (average of 9.8 years for patients with insomnia and 7.8 for those without insomnia). The third study, a hospital-based longitudinal Dutch study (PROPARK) assessed sleep quality in 412 patients (average PD duration of 10.6 years) with 27\% reporting insomnia; in cross-sectional analyses, these patients exhibited higher total motor UPDRS scores, and more motor complications and fluctuations [15]. 
Similar to the results presented here, an international multicenter study of 423 PD patients (mean PD duration of 6.7 years) reported no association between total motor UPDRS scores and concurrent EDS [20,37], but they did not report results for motor UPDRS sub-scores. In the PEG population-based patient cohort presented here, the only motor UPDRS sub-score associated with EDS was axial/gait/posture, which includes body bradykinesia; however, PIGD motor subtype was not associated with higher EDS symptoms. Only one other study, using a specialty clinic population from China, has reported positive associations between body bradykinesia scores and EDS [21]. Compared with this study's population, the Chinese sample had slightly shorter average PD duration (5.1 years) and scored higher on the total motor UPDRS.

Given these results, and as suggested previously by others [38], EDS, unlike insomnia, does not seem to be directly related to PD motor dysfunction resulting from primary nigrostriatal dopaminergic degeneration, but rather to features that suggest the degeneration of other neurotransmitter systems characteristic of more advanced disease [11]. For example, in postmortem and brain imaging studies in clinical samples [39], EDS has been related to the degeneration of the alertness system, including hypocretin neurons in the hypothalamus, noradrenergic neurons in the Locus Coeruleus, and serotonergic neurons in the Dorsal Raphe Nuclei. In this study's analyses, accounting for GDS scores in models for body bradykinesia and EDS moved the estimates towards no association, supporting the hypothesis [40] that depression in PD is associated with slowness of movement (body bradykinesia) and EDS; a future study will further explore the association of depression and sleep problems in the PEG cohort.

Therapy with levodopa and dopamine agonists is another factor frequently associated with sleep problems in PD in clinical practice. In general, the PEG study population seems to be under-treated, as almost one in 10 patients were not under any PD medication or treatment such as DBS [41], which could be credited to the study's source population being communities of mostly rural counties with intense agricultural activity, and low average education and income, where access to health care resources is often limited. Pharmacologically, it has been proposed that levodopa can affect circadian rhythms directly in PD patients through mechanisms that uncouple circadian rhythm and sleep regulation [34], such as altering melatonin secretion and action. In this study, levodopa doses (LED $\geq 500 \mathrm{mg} /$ day) were associated with both insomnia and EDS symptoms, in similar magnitude, while dopamine agonists doses (LED $\geq 200 \mathrm{mg} /$ day) did not affect sleep symptoms. Previous studies reported conflicting results regarding the contribution of levodopa therapy to sleep problems. A population-based study from Norway and a hospital-based Dutch study [19, 23, 24] found slightly higher LED in PD patients with EDS cross-sectionally, but LED did not predict worsening of EDS over time. The Norwegian ParkWest population-based cohort [19] reported no association of total LED and EDS for patients within five years from PD diagnosis, but a higher proportion of patients used dopamine agonists, $57 \%$ compared with $38 \%$ in our PEG cohort. Dopamine agonists have been shown to improve sleep in previous trials of rotigotine $[42,43]$, and to be associated with EDS and sleep-attacks in some clinical-based epidemiological studies [44], but not others [45]. No population-based studies addressed specific associations of dopamine agonists and sleep symptoms, and the present study may not have been able to detect associations if they are present, since only $38 \%$ of the study sample took such drugs.

Axial/gait/posture motor features of PD can be less responsive to dopaminergic therapy than tremor signs, which influence type and doses of PD medication prescribed [18]. Thus, to verify if these motor features can explain the association between levodopa and EDS, the models were 
further adjusted for axial/gait/posture motor sub-scores. Interestingly, this did not change the coefficients for levodopa (LED $\geq 500 \mathrm{mg}$ ), but the coefficient for the motor sub-score was no longer statistically significant. This may indicate that the association of axial/gait/posture sub-scores and EDS is mediated through levodopa doses.

As shown here and previously reported [15, 19, 20,46], non-motor and autonomic symptoms affect sleep quality and are related to EDS. Some of these manifestations can directly induce sleep fragmentation, such as nocturia. Others have been associated with circadian rhythms disruption, such as autonomic dysfunction. In fact, this study shows a strong association of sleep problems with autonomic symptoms including light headedness, constipation, drooling, chewing/swallowing, and urinary symptoms. This may not be surprising, as outputs from the suprachiasmatic nucleus, a structure in the hypothalamus recognized as the central pacemaker responsible for the regulation of circadian rhythms, innervates autonomous nervous system structures. Through these outputs, many independent circadian oscillators operate in peripheral organs and, coupled with hormonal secretion (involving melatonin and cortisol), they synchronize physiological functions, resulting, for example, in circadian fluctuations in blood pressure, urinary excretion and gastrointestinal activity. Degeneration of circadian system structures that induce circadian disruption would, thus, be manifesting in peripheral organs as autonomic dysfunction.

In the sub-group of 156 patients assessed twice for sleep problems during follow-up, insomnia scores did not worsen substantially, even when assessing subgroups with different PD severity (by UPDRS, PD duration, LED, and non-motor symptoms). On the other hand, EDS scores seem to worsen over time, especially in patients with worse PD severity at first follow-up, i.e., they reached the highest sleep score averages at their second follow-up. This may suggest that insomnia manifests early in the disease course, possibly concomitantly with worsening in tremor symptoms, but this sleep feature does not worsen as PD progresses. Two previous smaller studies examined progression of sleep symptoms in PD and also found insomnia prevalence to slightly decrease over follow-up time (8 years), while EDS worsened with PD progression [17, 19]. Future larger populationbased longitudinal cohort studies of PD are still necessary to address how circadian dysfunction progresses in PD clinical course.

Lack of temporality is a limitation of this study. The main results reported are cross-sectional, while the exposures and outcomes studied are part of a vicious cycle of deterioration during PD progression and influence each other longitudinally. The associations reported here refer to prevalent sleep symptoms at on average six years after PD diagnosis, and we do not know when these sleep problems started. Another potential limitation refers to the absence of objective measures of sleep quality and structure, since we relied solely on self-reported information. This is, however, a problem common to all population-based studies with large numbers of patients, due to feasibility constraints that do not support using objective sleep laboratory assessments, such as polysomnography. Unmeasured residual confounding is unavoidable in observational research, however, the regression models took into account explicit hypothesized causal structures [33], different from purely predictive modeling approaches of insomnia and EDS in PD. Important strengths of this study are the large number of subjects and the population-based approach to identify PD cases, which likely yields estimates that are more representative of all PD patients, than those based on very selected clinic-based patient samples. 


\section{Conclusions}

In conclusion, this study provides epidemiological evidence that motor and non-motor dysfunction in PD is associated with insomnia and EDS symptoms, but specific features and level of severity affect these sleep symptoms differently. While motor severity measured by total UPDRS score and sub-scores of tremor, limb bradykinesia and motor complications mainly impact insomnia, EDS symptoms were related to axial/gait/posture motor features, which may indicate higher levodopa doses and/or more advanced disease. Future assessments of sleep problems in longitudinal population-based studies are needed to help improve patients' overall health-related quality of life.

\section{Acknowledgments}

We acknowledge the contributions of Dr Yvette Bordelon for her work as a Movement Disorders specialist conducting clinical assessments for the study. We would also like to thank all patients with Parkinson's disease and their caregivers, and all the PEG Study staff, for their time and their efforts that made this research possible.

\section{Additional Materials}

The following additional materials are uploaded at the page of this paper.

1. Figure S1: Proposed Directed Acyclic Graph (DAG) depicting hypothesized relation of factors considered in cross-sectional analyses. Arrows in purple represent the main potential causal relations we aimed at estimating. Variables in parenthesis are not measured. UPDRS-III= measure of motor dysfunction severity. EDS= excessive daytime sleepiness.

2. Figure S2: Correlations among PD-related and sleep variables considered in analyses.

\section{Author Contributions}

Jeff M Bronstein and Adrienne Keener conducted most of the clinical assessments of Parkinson's disease patients, at baseline and follow-up. Aline Duarte Folle conducted the analyses, with support from Kimberly Paul and Cynthia Kusters, and wrote the first draft of the paper, under supervision of Beate Ritz, who is responsible for the conception of the study. All authors contributed in discussing results and editing the manuscript.

\section{Funding}

This research study has been funded by the National Institute of Environmental Health Sciences of the National Institutes of Health (grants numbers: R01 ES010544, U54-ES012078, P01-ES016732, P50-NS038367, and initial pilot funding P30- ES07048), and by the American Parkinson's disease Association (grant number 20161386).

\section{Competing Interests}

The authors have declared that no competing interests exist. 


\section{References}

1. Parkinson J. An essay on the shaking palsy. J Neuropsychiatry Clin Neurosci. 2002; 14: 223-236.

2. Mouret J. Differences in sleep in patients with Parkinson's disease. Electroencephalogr Clin Neurophysiol. 1975; 38: 653-657.

3. Tandberg E, Larsen JP, Karlsen K. A community-based study of sleep disorders in patients with Parkinson's disease. Mov Disord. 1998; 13: 895-899.

4. Chaudhuri KR, Pal S, Dimarco A, Whately-Smith C, Bridgman K, Mathew R, et al. The Parkinson's disease sleep scale: A new instrument for assessing sleep and nocturnal disability in Parkinson's disease. J Neurol Neurosurg Psychiatry. 2002; 73: 629-635.

5. Videnovic A, Högl B. Disorders of sleep and circadian rhythms in Parkinson's disease. 1st ed. Vienna: Springer Vienna; 2015.

6. Suzuki K, Miyamoto M, Miyamoto T, Hirata K. Restless legs syndrome and leg motor restlessness in Parkinson's disease. Parkinsons Dis. 2015; 2015: 490938.

7. Li S, Wang Y, Wang F, Hu LF, Liu CF. A new perspective for Parkinson's disease: Circadian rhythm. Neurosci Bull. 2016; 33: 62-72.

8. Fifel K. Alterations of the circadian system in Parkinson's disease patients. Mov Disord. 2017; 32: 682-692.

9. Avidan A, Hays RD, Diaz N, Bordelon Y, Thompson AW, Vassar SD, et al. Associations of sleep disturbance symptoms with health-related quality of life in Parkinson's disease. J Neuropsychiatry Clin Neurosci. 2013; 25: 319-326.

10. Xu W, Tan CC, Zou JJ, Cao XP, Tan L. Sleep problems and risk of all-cause cognitive decline or dementia: An updated systematic review and meta-analysis. J Neurol Neurosurg Psychiatry. 2019; 91: 1-9.

11. Stefani A, Högl B. Sleep in Parkinson's disease. Neuropsychopharmacology. 2020; 45: 121-128.

12. Daroff RB. The international classification of sleep disorders: Diagnostic and coding manual. Chest. 1991; 41: 160-160.

13. Marinus J, Visser M, Van Hilten JJ, Lammers GJ, Stiggelbout AM. Assessment of sleep and sleepiness in Parkinson disease. Sleep. 2003; 26: 1049-1054.

14. Sateia MJ. International classification of sleep disorders-third edition highlights and modifications. Chest. 2014; 146: 1387-1394.

15. Zhu K, van Hilten JJ, Marinus J. The course of insomnia in Parkinson's disease. Parkinsonism Relat Disord. 2016; 33: 51-57.

16. Porter B, MacFarlane R, Walker R. The frequency and nature of sleep disorders in a communitybased population of patients with Parkinson's disease. Eur J Neurol. 2008; 15: 46-50.

17. Gjerstad MD, Wentzel-Larsen T, Aarsland D, Larsen JP. Insomnia in Parkinson's disease: Frequency and progression over time. J Neurol Neurosurg Psychiatry. 2007; 78: 476-479.

18. Ratti PL, Negre-Pages L, Perez-Lloret S, Manni R, Damier P, Tison F, et al. Subjective sleep dysfunction and insomnia symptoms in Parkinson's disease: Insights from a cross-sectional evaluation of the French CoPark cohort. Park Relat Disord. 2015; 21: 1323-1329.

19. Tholfsen LK, Larsen JP, Schulz J, Tysnes OB, Gjerstad MD. Development of excessive daytime sleepiness in early Parkinson disease. Neurology. 2015; 85: 162-168. 
20. Amara AW, Chahine LM, Caspell-Garcia C, Long JD, Coffey C, Högl B, et al. Longitudinal assessment of excessive daytime sleepiness in early Parkinson's disease. J Neurol Neurosurg Psychiatry. 2017; 88: 653-662.

21. Xiang $Y, X u Q$, Sun $Q$, Wang ZQ, Tian $Y$, Fang $L$, et al. Clinical features and correlates of excessive daytime sleepiness in Parkinson's disease. Front Neurol. 2019; 10: 1-9.

22. Junho BT, Kummer A, Cardoso F, Teixeira AL, Rocha NP. Clinical predictors of excessive daytime sleepiness in patients with Parkinson's disease. J Clin Neurol. 2018; 14: 530-536.

23. Tandberg E, Larsen JP, Karlsen K. Excessive daytime sleepiness and sleep benefit in Parkinson's disease: A community-based study. Mov Disord. 1999; 14: 922-927.

24. Zhu K, van Hilten JJ, Marinus J. Course and risk factors for excessive daytime sleepiness in Parkinson's disease. Parkinsonism Relat Disord. 2016; 20: 980-985.

25. Duarte Folle A, Paul KC, Bronstein JM, Keener AM, Ritz B. Clinical progression in Parkinson's disease with features of REM sleep behavior disorder: A population-based longitudinal study. Parkinsonism Relat Disord. 2019; 62: 105-111.

26. Wang A, Costello S, Cockburn M, Zhang X, Bronstein J, Ritz B. Parkinson's disease risk from ambient exposure to pesticides. Eur J Epidemiol. 2011; 26: 547-555.

27. Ritz B, Rhodes SL, Bordelon $\mathrm{Y}$, Bronstein J. $\alpha$-Synuclein genetic variants predict faster motor symptom progression in idiopathic Parkinson disease. PLoS One. 2012; 7: e36199.

28. Keener AM, Paul KC, Folle A, Bronstein JM, Ritz B. Cognitive impairment and mortality in a population-based Parkinson's disease cohort. J Parkinsons Dis. 2018; 8: 353-362.

29. Tomlinson CL, Stowe R, Patel S, Rick C, Gray R, Clarke CE. Systematic review of levodopa dose equivalency reporting in Parkinson's disease. Mov Disord. 2010; 25: 2649-2653.

30. Stebbins GT, Goetz CG, Burn DJ, Jankovic J, Khoo TK, Tilley BC. How to identify tremor dominant and postural instability/gait difficulty groups with the movement disorder society unified Parkinson's disease rating scale: Comparison with the unified Parkinson's disease rating scale. Mov Disord. 2013; 28: 668-670.

31. Höglund A, Hagell P, Broman J-E, Pålhagen S, Sorjonen K, Fredrikson S. A 10-year follow-up of excessive daytime sleepiness in Parkinson's disease. Park Dis. 2019; 2019: 5708515.

32. Chaudhuri KR, Pal S, DiMarco A. The Parkinson's disease sleep scale: A new instrument for assessment of sleep, nocturnal disability and daytime sleepiness in Parkinson's disease. J Neurol Neurosurg Psychiatry. 2002; 73: 629-635.

33. Greenland S, Pearl J, Robins JM. Causal diagrams for epidemiologic research. Epidemiology. 1999; 10: 37-48.

34. Mantovani S, Smith SS, Gordon R, O'Sullivan JD. An overview of sleep and circadian dysfunction in Parkinson's disease. J Sleep Res. 2018; 27: e12673.

35. French IT, Muthusamy KA. A review of sleep and its disorders in patients with Parkinson's disease in relation to various brain structures. Front Aging Neurosci. 2016; 8: 1-17.

36. Bhidayasiri R, Sringean J, Trenkwalder C. Mastering nocturnal jigsaws in Parkinson' s disease: A dusk-to-dawn review of night-time symptoms. J Neural Transm. 2020; 127: 763-777.

37. Simuni T, Caspell-Garcia C, Coffey C, Chahine LM, Lasch S, Oertel WH, et al. Correlates of excessive daytime sleepiness in de novo Parkinson's disease: A case control study. Mov Disord. 2015; 30: 1371-1381.

38. Höglund A, Broman J-E, Pålhagen S, Fredrikson S, Hagell P. Is excessive daytime sleepiness a separate manifestation in Parkinson's disease? Acta Neurol Scand. 2015; 132: 97-104. 
39. Wilson H, Giordano B, Turkheimer FE, Chaudhuri KR, Politis M. Serotonergic dysregulation is linked to sleep problems in Parkinson's disease. Neurolmage Clin. 2018; 18: 630-637.

40. Rampello L, Chiechio S, Raffaele R, Vecchio I, Nicoletti F. The SSRI, citalopram, improves bradykinesia in patients with Parkinson's disease treated with L-dopa. Clin Neuropharmacol. 2002; 25: 21-24.

41. Kang GA, Bronstein JM, Masterman DL, Redelings M, Crum JA, Ritz B. Clinical characteristics in early Parkinson's disease in a central California population-based study. Mov Disord. 2005; 20: 1133-1142.

42. Vallderiola F, Compta Y, Aparicio J, Tarradellas J, Salazar G, Oliver JM, et al. Effects of night-time use of rotigotine on nocturnal symptoms in Parkinson's disease. Parkinsons Dis. 2015; 2015: 475630.

43. Pierantozzi $M$, Placidi $F$, Liguori $C$, Albanese $M$, Imbriani $P$, Marciani $M G$, et al. Rotigotine may improve sleep architecture in Parkinson's disease: A double-blind, randomized, placebocontrolled polysomnographic study. Sleep Med. 2016; 21: 140-144.

44. Homann CN, Wenzel K, Suppan K, Ivanic G, Kriechbaum N, Crevenna R, et al. Sleep attacks in patients taking dopamine agonists: Review. BMJ. 2002; 324: 1483-1487.

45. Ataide M, Franco CMR, Lins OG. Daytime sleepiness in Parkinson's disease: Perception, influence of drugs, and mood disorder. Sleep Disord. 2014; 2014: 1-5.

46. Matsubara $T$, Suzuki K, Fujita $H$, Watanabe $Y$, Sakuramoto $H$, Matsubara $M$, et al. Autonomic symptoms correlate with non-autonomic non-motor symptoms and sleep problems in patients with Parkinson's disease. Eur Neurol. 2018; 80: 193-199.

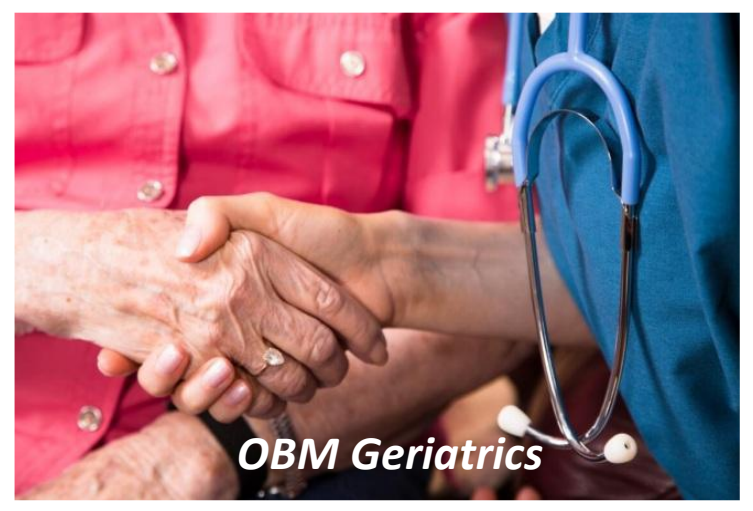

Enjoy OBM Geriatrics by:

1. Submitting a manuscript

2. Joining in volunteer reviewer bank

3. Joining Editorial Board

4. Guest editing a special issue

For more details, please visit:

http://www.lidsen.com/journals/geriatrics 